\title{
The low barrier methyl internal rotation in the rotational spectrum of 3-methylphenylacetylene
}

\author{
Daniel A. Obenchain ${ }^{\mathrm{a}}$, Pablo Pinacho ${ }^{\mathrm{a}}$, Sabrina Zinn ${ }^{\mathrm{a}}$, Melanie Schnell ${ }^{\mathrm{a}, \mathrm{b}}$ \\ ${ }^{a}$ Deutsches Elektronen-Synchrotron, Notkestr. 85, D-22607 Hamburg, Germany \\ ${ }^{b}$ Institute of Physical Chemistry, Christian-Albrechts-Universität zu Kiel, Max-Eyth-Strasse \\ 1, D-24118 Kiel, Germany
}

\begin{abstract}
The rotational spectrum of 3-methylphenylacetylene has been recorded in the 2$8 \mathrm{GHz}$ region using a broadband chirped-pulse microwave spectrometer. Torsionrotation transition splittings are observed from a tunneling motion along the methyl internal rotation axis. The XIAM program was used to characterize the splitting, yielding an internal rotation barrier, $V_{3}$, of $11.21745 \pm 0.00002 \mathrm{~cm}^{-1}$. While this barrier is considered low, fits of $A$-state only transitions yield a quality, rigid-rotor fit and are compared to the combined $A / E$ fits. Computationally predicted barriers are estimated between 14.4 and $28.9 \mathrm{~cm}^{-1}$.
\end{abstract}

Keywords: Low barrier, internal rotation, rotational spectroscopy, 3mp, 3-methylphenylacetylene, 3-ethynyltoluene, CAS 766-82-5

\section{Introduction}

The handling of low barrier methyl internal rotation in high-resolution spectroscopy remains a challenging aspect to spectroscopic research. As was outlined recently by Eibl et al., [1] the research challenges of low barrier $\left(V_{3}>30\right.$ ${ }_{5} \mathrm{~cm}^{-1}$ ) internal rotation are a combination of experimental and computational shortcomings. Computationally, low barriers are difficult to predict to a level of confidence that is useful to experimentalists, which increases the difficulty of

\footnotetext{
*Please address correspondence to Melanie Schnell

Email address: melanie.schnell@desy.de (Melanie Schnell)
} 
assigning observed spectra based on these predictions. Even with more accurate predictions, the large splittings from low barriers still present a challenge in assignment for experimentalists, and splittings can be on the order of hundreds of $\mathrm{MHz}$ or even GHz. Additionally, the fitting procedure is often complicated by the need for large amounts of tunneling parameters, and there is not a well established procedure for determining which tunneling parameters will be important in the fitting process. Often, the intial values of the tunneling parameters are difficult to predict. Unlike centrifugal distortion constants, the physical meaning of the torsional tunneling parameters is not as clear.

Despite the difficulties associated with their assignment in rotational spectroscopy, internal rotation barriers are chemically useful as they can serve as 20 a probe for local and long-range interactions in a molecule. When following a specific chemical motif, the internal rotation barrier can be used as a benchmark data set for computational methods. An example is seen in the change of the potential barrier of toluene for various levels of halogenation around the benzyl ring. In toluene, the $V_{6}$ barrier is reported as $4.837920599(11) \mathrm{cm}^{-1},[2]$ and increases for $p$-chlorotoluene $\left(4.872(14) \mathrm{cm}^{-1}\right)[3$, 3,5-difluorotoluene $(7.156(84)$ $\left.\mathrm{cm}^{-1}\right)$ [4 and 2,4-difluorotoluene $\left(12.432(20) \mathrm{cm}^{-1}\right)$, 4] but decreases slightly for $p$-fluorotoluene $\left(4.8298(64) \mathrm{cm}^{-1}\right)[5]$. In a more recent study, an investigation of $p$-halotoluenes found a $p$-chlorotoluene $V_{6}$ barrier of $4.836(29) \mathrm{cm}^{-1}$, which was also found to be a good barrier to describe the tunneling motion in so $p$-bromotoluene and $p$-iodotoluene. $[6$. These studies provide a good data set for the interplay of local steric interactions and ring induction effects as measured by the change in internal rotation barrier.

An example of the change in internal rotation barrier in van der Waals com35 plexes can also be seen, again with toluene as the subject. In a rotational spectroscopy study of the toluene complex with $\mathrm{SO}_{2}$, the potential barrier was increased to $V_{3}=83.236(2) \mathrm{cm}^{-1}$. [7] Comparing this to a non-polar binding partner, the potential barrier in the Ar...toluene complex was determined to 
be $20 \pm 1 \mathrm{~cm}^{-1}$ in an 2D laser induced flu orescence experiment. 8 . In both

cases, the interaction of the binding partner is with the $\pi$ cloud of the ring, and changes in the barrier are likely a combination of intermolecular forces and steric effects. It should be noted that from a change of symmetry, the barriers are now 3 -fold in the van der Waals complexes, instead of the 6 -fold in the toluene and $p$-toluene molecules.

45

In a similar fashion to the toluene studies, 3-methylphenylacetelyne (3mp, Figure 1) can be used as a basic structural motif to measure changes in the methyl group internal rotation barrier due to changes in the local chemical environment. Unlike with toluene, van der Waals complexes involving $3 \mathrm{mp}$ can also have interactions with the carbon-carbon triple bond, instead of the $\pi$ cloud of the ring. This will remove some of the steric interactions on the internal rotation barrier that are caused directly by forming a gas-phase complex, instead allowing for changes in barrier height from dispersion interactions and from ring induction induced by dispersion interactions to be observed. To begin this series, this study focuses on the internal rotation of the low barrier internal methyl rotation of the $3 \mathrm{mp}$ monomer.

\section{Experimental Methods}

The broadband rotational spectrum from $2-8 \mathrm{GHz}$ was collected on the COMPACT spectrometer at the Deutsches Elektronen-Synchrotron in Hamburg, Germany. The details of the setup are described elsewhere, [9, 10] and is based on the chirp-pulsed broadband microwave (CP-FTMW) spectrometer design of Pate and co-workers. 11] A sample of 3mp was obtained from SigmaAldrich $(97 \%)$ and used without further purification. With a boiling point of $170-175^{\circ} \mathrm{C}$, a sufficient vapor pressure was achieved at room temperature. The vapor of $3 \mathrm{mp}$ was seeded in 4 bar (absolute pressure) of 5.0 neon and expanded through a General Valve Series 9 nozzle with an orifice of $1.1 \mathrm{~mm}$ at a repetition 
rate of eight $\mathrm{Hz}$. For each gas pulse, eight direct excitation pulses of $2-8 \mathrm{GHz}$ were used. The free-induction decay of the induced polarization was directly detected via a microwave horn antenna, resulting in an effective experimental repetition rate of $64 \mathrm{~Hz}$. A total of one million free-induction decay signals were collected and converted to the frequency domain using a fast-Fourier transformation. Fitting procedures utilized the SPFIT/SPCAT software of Pickett [12] for initial fits. The two tunneling states, $A$ and $E$, produced by the coupling of the methyl rotor angular momentum to the rotational angular momentum, were predicted using the XIAM program 13 . While there are cases of SPFIT/SPCAT being used to predict and fit the $A / E$ tunneling states and torsional splittings of a methyl rotor, XIAM presents a more straightforward approach to the handling of the torsional splitting. XIAM uses the inertial axis method to treat internal rotation, and examples of its use are numerous. The graphical interface program AABS 14 was used to aid in pattern matching from predictions to assignments. Links to all programs used can be found on the PROSPE website, 15] which includes examples and references their respective uses.

\section{Computational Methods}

All calculations were completed using the ORCA computational chemistry software version 4.1.0 16] on the Maxwell Cluster at Deutsches ElektronenSynchrotron. Optimizations of 3mp were completed using three different combinations of methods and basis sets. First, the single hybrid DFT, B3LYP, 17 with a def2-SVDP [18] basis set was employed. A double hybrid DFT level of theory was also used, the B2PLYP 19] method with a def2-TZVPP 20] basis set. Both density functional methods were applied using Grimme's dispersion correction, D3(BJ). 21, 22] Finally, an MP2[23] calculation with the aug-ccpVDZ 24, 25] basis set was employed. Once the structure of 3mp was optimized for each method, that structure was used in a relaxed scan calculation of the methyl groups, to predict the $V_{3}$ potential barrier, at increments of $10^{\circ}$ from $0^{\circ}$ to $120^{\circ}$. The optimized equilibrium state rotational constants and predicted $V_{3}$ 


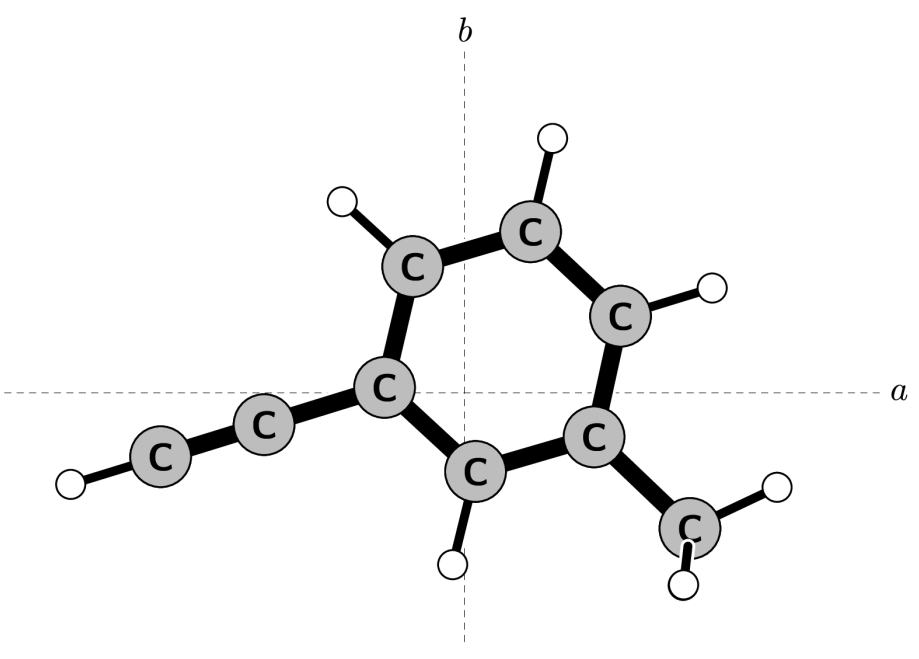

Figure 1: Optimized structure of 3mp from the B2PLYP/def2-TZVPP D3(BJ) calculation.

barriers can be found in Table 1 and the optimized structure form the B2PLYP calculation is shown in Figure 1. The predicted value of $\delta$, the angle between the methyl internal rotation axis and the principal $a$-axis, can also be found in Table 1.

\section{Results and Discussion}

Rotational patterns belonging to the $a$-type transitions of $3 \mathrm{mp}$ were easily identified from predicted rotational constants, consistent with the approximately $1 \mathrm{D} \mu_{a}$-dipole component predicted from the optimized structures in ${ }_{105}$ Table 1. Initial fits of the $A$-state were completed using SPFIT/SPCAT. The $A$-symmetry torsional sub-level of the methyl tunneling states can often be described by a rigid-rotor Hamiltonian, which is why it was fit before the $E$ symmetry sub-level. In specific cases, the $E$-state transitions can also be fit separate of the $A$-state 26, 27] but this has only been carried out for systems with an internal rotation barrier above $400 \mathrm{~cm}^{-1}$. [26, 27, 28, While there is a small $\mu_{b}$ dipole moment component predicted for $3 \mathrm{mp}$, only $a$-type transitions 
Table 1: Predicted spectroscopic constants for 3mp.

\begin{tabular}{clccc}
\hline \hline & & $\begin{array}{c}\text { B3LYP } \\
\text { def2-SVPD }\end{array}$ & $\begin{array}{c}\text { B2PLYP } \\
\text { def2-TZVPP }\end{array}$ & $\begin{array}{c}\text { MP2 } \\
\text { aug-cc-pVDZ }\end{array}$ \\
\hline$A$ & $\mathrm{MHz}$ & 3280 & 3313 & 3243 \\
$B$ & $\mathrm{MHz}$ & 1186 & 1197 & 1171 \\
$C$ & $\mathrm{MHz}$ & 876 & 884 & 865 \\
$V_{3}$ & $\mathrm{~cm}^{-1}$ & 28.9 & 14.4 & 14.7 \\
$V_{3}$ & $\mathrm{~kJ} / \mathrm{mol}$ & 0.346 & 0.172 & 0.176 \\
$\delta^{\mathrm{a}}$ & $\mathrm{deg}$ & 43.0 & 43.1 & 43.2 \\
$\mu_{a}$ & $\mathrm{D}$ & 1.01 & 1.00 & 0.94 \\
$\mu_{b}$ & $\mathrm{D}$ & -0.07 & -0.07 & -0.10 \\
$\mu_{c}{ }^{\mathrm{b}}$ & $\mathrm{D}$ & 0 & 0 & 0 \\
\hline \hline
\end{tabular}

a $\delta$ is the angle between the methyl rotor and the $a$-axis.

$\mathrm{b}$ The dipole projection on the $c$-axis is zero by definition as the molecule has a plane of symmetry in the $a b$-plane. 
were observed for the $A$-state. The initial fits using $A$-state rotational constants and the barrier and methyl rotation angle parameter $\delta$ from the B2PLYP optimized structure were used to predict transitions belonging to the $E$-state. Finally, a total of $19 A$-state transitions and $35 E$-state transitions were observed. A region of the recorded spectrum showing the $A / E$ torsional splittings is shown in Figure 2 Searches for the singly substituted ${ }^{13} \mathrm{C}$ isotopologues in natural abundances were unsuccessful. A few possible candidates were identified for some of the stronger $a$-type transitions, but not enough were found to make a fit of the isotopic species.

A fit of $A$-state transitions with only $K_{a}=0,1$ yields a satisfactory rigidrotor fit with an rms value of $8.8 \mathrm{kHz}$, a typical rms for a molecule with no large amplitude motions when using the Hamburg COMPACT spectrometer. ${ }_{125}$ Inclusion of higher $K_{a}$ transitions from the $A$-state, up to $K_{a}=3$, does not significantly increase the uncertainty of the fit, now $15.0 \mathrm{kHz}$. This has the most prominent effect on the $A$ constant, changing by hundreds of $\mathrm{kHz}$ with the addition of higher $K_{a}$ transitions. However, the inclusion of centrifugal distortion constants does not significantly affect the quality of the fit. Various combinations of the five quartic centrifugal distortion constants showed that only $D_{J K}$ made any significant contribution, and $D_{J}$ often had the incorrect sign when it was included ( $D_{J}$ must be positive). With these dubious values, it was assumed that the contributions of the distortion constants were compensating for the tunneling parameters in the $A$-state only fit, and therefore only the rigid-rotor constants are presented in Table 2 .

Using the XIAM program, and the A-state fit as a starting point, the Estate lines were predicted and fit. The value of $V_{3}=14.4 \mathrm{~cm}^{-1}$ was taken from the B2PLYP calculation as an initial value for the prediction, although starting 140 from either the B3LYP $\left(28.9 \mathrm{~cm}^{-1}\right)$ or MP2 $\left(14.7 \mathrm{~cm}^{-1}\right)$ calculation yields the same result. As seen in Table 1, the internal rotation structural parameter $\delta$, the angle between the internal rotation axis and $a$-axis, did not vary between the 
three computational methods. The other structural internal rotation parameter, $\epsilon$, was fixed to zero. This parameter is the angle between the projection of the methyl axis onto the $b c$-plane and the $b$-axis, but as the molecule is heavy-atom planar with the methyl internal rotation axis and the $b$-axis coplanar, $\epsilon$ is zero.

Inclusion of the tunneling parameters from XIAM was essential to producing the fit found in Table 2. The choice of parameters to include in the $A / E$ state fits with XIAM required careful consideration of parameter values and correlations between these parameters. Due to the low number of $E$-state transitions, $V_{3}$ and $F$ could not be fit simultaneously, and $F$ was fixed at the values of $158.3 \mathrm{GHz} . \quad F$ is the angular momentum term for the methyl top, and it is common that it correlates to $V_{3}$ during the fitting process. Often it is chosen to be fixed in favor of the $V_{3}$ term. The internal rotation-molecular rotation distortion tunneling parameters, $D_{c 3 J}, D_{\pi 2 J}, D_{\pi 2 K}$, and $D_{\pi 2-}$, were included, although the $D_{c 3 J}$ parameter was the most significant in reducing the error of the fit and in including the $b$-, $c$-, and $x$-type selection rule transitions of the $E$-state. The $c$-type transitions would be dipole forbidden if the molecule did not have methyl torsional coupling, but the large angular momentum coupling from the methyl torsion in the $E$-state allows for the observation of the $c$-type and $x$-type transitions. At various points in the fitting procedure, the inclusion of the centrifugal distortion constants did little for the quality of the fit, and they were highly correlated to each other and to other parameters, so that they were again excluded from the fit. Attempts were made to fit a $V_{6}$ potential, and a value of about $2 \mathrm{~cm}^{-1}$ was determined, but this was highly correlated to the $V_{3}$ value. No matter which combination of tunneling parameters were used, the potential barrier $V_{3}$ was consistently around $11.2 \mathrm{~cm}^{-1}$, imparting credibility to the value of $11.21745(2) \mathrm{cm}^{-1}$ in Table 2. In the fit chosen for Table 2 , there is 170 still high correlation between the $D_{c 3 J}$ and $D_{\pi 2 J}$.

An improved fit uncertainty can be achieved by removing certain $E$-state transitions form the fit. However, this keeps the $V_{3}$ close to the 11.21745(2) 


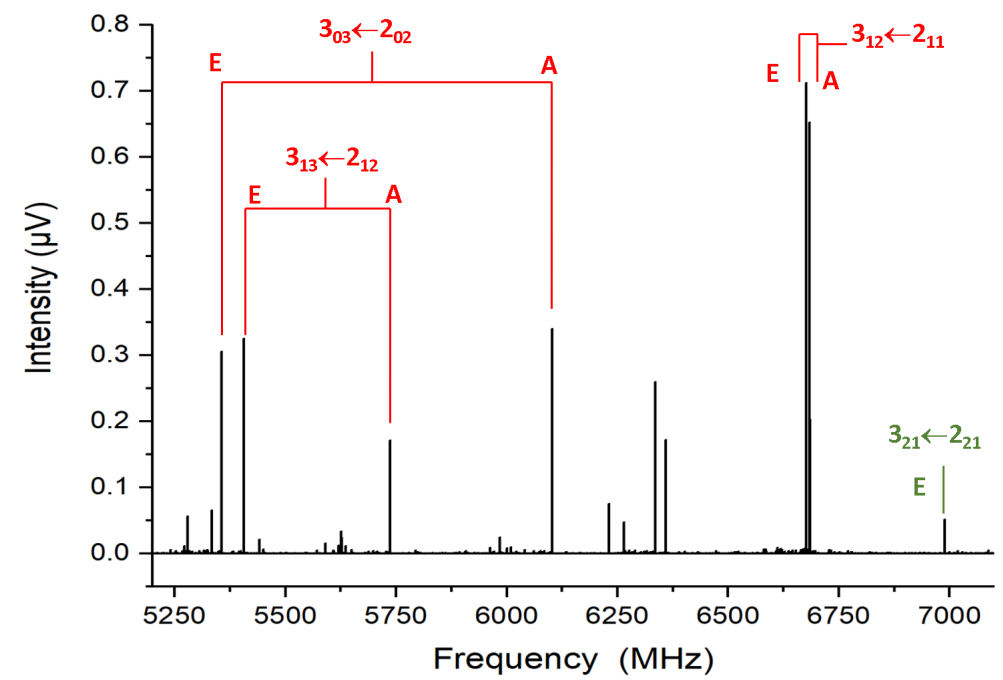

Figure 2: Observed spectrum of $3 \mathrm{mp}$ from $5200 \mathrm{MHz}$ to $7100 \mathrm{MHz}$. Red labeled transitions show the $A / E$ symmetry state splittings for selected $a$-type transitions. $A / E$ splittings vary depending on transition, from a few $\mathrm{MHz}$ to almost a GHz. In green, an $x$-type transition belonging to the $E$-state. Many of the weaker transitions between 5250-5750 are $c$-type and $x$-type transitions. Not seen in this frequency region are the observed $b$-type transitions. 
Table 2: Results from the spectroscopic fits of SPFIT and XIAM for 3mp

\begin{tabular}{|c|c|c|c|c|}
\hline & & SPFIT & SPFIT & Xiam \\
\hline \multirow{2}{*}{ State } & & $A$ only & $A$ only & \multirow{2}{*}{$A \& E$} \\
\hline & & $K_{a}=0$ and $K_{a}=1$ & all $K_{a}$ & \\
\hline$A$ & $\mathrm{MHz}$ & $3330.32(13)^{\mathrm{a}}$ & $3330.7009(94)$ & $3294.467(26)$ \\
\hline$B$ & $\mathrm{MHz}$ & $1196.7401(11)$ & $1196.73760(98)$ & $1192.6675(18)$ \\
\hline$C$ & $\mathrm{MHz}$ & $880.35776(72)$ & $880.35771(98)$ & $880.3578(18)$ \\
\hline$D_{c 3 J}$ & $\mathrm{MHz}$ & - & - & $-2.04(14)$ \\
\hline$D_{\pi 2 J}$ & $\mathrm{MHz}$ & - & - & $0.145(12)$ \\
\hline$D_{\pi 2 K}$ & $\mathrm{MHz}$ & - & - & $0.7266(14)$ \\
\hline$D_{\pi 2-}$ & $\mathrm{MHz}$ & - & - & $-0.00268(33)$ \\
\hline$F$ & $\mathrm{GHz}$ & - & - & {$[158.3]^{\mathrm{b}}$} \\
\hline$V_{3}$ & $\mathrm{~cm}^{-1}$ & - & - & $11.21745(2)^{\mathrm{c}}$ \\
\hline$\delta^{\mathrm{d}}$ & $\operatorname{deg}$ & - & - & $43.06908(14)$ \\
\hline$N^{\mathrm{e}}$ & & 11 & 19 & 54 \\
\hline$r m s^{\mathrm{f}}$ & $\mathrm{kHz}$ & 8.8 & 15.0 & 29.9 \\
\hline
\end{tabular}

${ }^{\text {a }}$ Values in parenthesis are $1 \sigma$ uncertainties in the least significant digits.

b Value was fixed during fitting.

${ }^{c}$ For $V_{3}$, the value in parenthesis is the absolute error in the least significant digit.

$\mathrm{d} \delta$ is the angle between the methyl rotor and the $a$-axis.

e The number of transitions used in the fit.

f The uncertainty of the fit. 
$\mathrm{cm}^{-1}$ value of the fit of all transitions. As this does not change the focus parameter of this study, $V_{3}$, all measured transitions were included in the final fit. However, it is clear that there are missing tunneling parameters needed to describe the internal rotation in 3mp. XIAM does not contain any additional parameters that were not tried in the fitting procedure. Other programs that can fit methyl internal rotation were also used, and these programs have additional tunneling parameters. An attempt was made with Groner's ERHAM 29, 30, to find a more accurate fit, but as is stated in the literature, "ERHAM requires some expertise in choosing the best set of parameters," 31 and a converged fit could not be obtained for 3mp, even with the excellent list of tunneling parameters provided in one of more recent ERHAM publications from the author. 30. An attempt to fit the observed data set with Ilyushin's RAM36 2] program produced high uncertainties in the fit, but this is likely do to the incorrect choice of tunneling parameters. Of the three internal rotation fitting programs tried, we were only able to obtain a quality fit with XIAM. Likely this is a mixture of faults, first with the limited data set. The majority of the observed transitions are low $J$ and low $K$, as the molecule is not heavy with only 9 heavy atoms. Likely measurements at higher frequency ranges would be needed to explore the use of additional tunneling parameters in ERHAM and RAM36. Even then, the choice of tunneling parameters in these programs is not always obvious, and careful consideration will be needed in choosing the correct terms.

195

Comparison of the observed internal rotation barrier, $V_{3}=11.21745(2)$ $\mathrm{cm}^{-1}$, to the predicted barriers of the computational methods does shed some insight onto which methods may perform better at predicting low barrier internal rotation problems. Both the double-hybrid B2PLYP and the MP2 calculations predicted a barrier height to within $3.5 \mathrm{~cm}^{-1}$ of the experimental value. The B3LYP calculation is significantly less accurate, over-predicting by $17.7 \mathrm{~cm}^{-1}$, an error of $61 \%$. In this case, the more Hartee-Fock included in the calculation, the more accurate the resulting barrier. However, this is a comparison of only one experimental result with one set of computational methods. A more accu- 
rate comparison would come from either an experimental data set including the para and ortho equivalent methylphenylacetelyne molecules, or a set of van der Waals complexes to show the effect of the intermolecular forces on the internal rotation barrier, as was pointed out in the essay of Mata and Suhm. 32.

\section{Conclusion}

With an understanding of the potential barrier, van der Waals complexes with $3 \mathrm{mp}$ can now be studied to observe indirectly the effects of intermolecular forces. Indeed, some complexes have already been observed that have no steric interaction with the methyl rotor, but still affect the barrier to internal rotation. 33] It is believed that with an increased mass and lower rotational constants of the complexes of $3 \mathrm{mp}$ compared to the monomer, additional transitions will allow for a better determination of the tunneling parameters needed to describe the internal rotation motion in $3 \mathrm{mp}$.

\section{Supplementary Information}

Input and output files for the SPFIT/SPCAT and the XIAM fits presented in Table 2 are included in the supplementary material.

\section{Acknowledgements}

Impetuses for the larger series of 3-methylphenylacetlyene van der Waals complex studies was provided by Dr. Ginny Karir and Prof. Dr. Martin Suhm as part of the DFG-SPP 1807 Program collaboration, and it was supported by the SPP 1807 grant number SCHN1280/4-2. This research was supported in part through the European XFEL and DESY funded Maxwell computational resources operated at Deutsches Elektronen-Synchrotron (DESY), Hamburg, Germany. PP would specifically like to thank the Alexander von Humboldt 
Foundation for funding.

\section{References}

[1] K. Eibl, R. Kannengießer, W. Stahl, H. V. L. Nguyen, I. Kleiner, 235 Low barrier methyl rotation in 3-pentyn-1-ol as observed by microwave spectroscopy, Molecular Physics 114 (23) (2016) 3483-3489. doi:10.1080/00268976.2016.1239034. URL https://www.tandfonline.com/doi/full/10.1080/00268976. 2016.1239034

[2] V. V. Ilyushin, Z. Kisiel, L. Pszczólkowski, H. Mäder, J. T. Hougen, A new torsion-rotation fitting program for molecules with a sixfold barrier: Application to the microwave spectrum of toluene, Journal of Molecular Spectroscopy 259 (1) (2010) 26-38. doi:10.1016/j.jms.2009.10.005.

[3] G. E. Herberich, Mikrowellenspektrum, Hinderungspotential der internen Rotation und teilweise r0-Struktur des para-Chlortoluols, Zeitschrift fur Naturforschung - Section A Journal of Physical Sciences 22 (5) (1967) 761764. doi:10.1515/zna-1967-0524.

[4] K. P. Rajappan Nair, M. K. Jahn, A. Lesarri, V. V. Ilyushin, J. U. Grabow,

n Six-fold-symmetry internal rotation in toluenes: the low barrier challenge of 2,6- and 3,5-difluorotoluene, Physical Chemistry Chemical Physics 17 (39) (2015) 26463-26470. doi:10.1039/c5cp03751d.

URL www.rsc . org/pccp

[5] J. Rottstegge, H. Hartwig, H. Dreizler, The rotational spectrum, structure and barrier V6 to internal rotation of p-fluorotoluene, Journal of Molecular Structure 478 (1-3) (1999) 37-47. doi:10.1016/S0022-2860(98)00656-5.

[6] V. A. Shubert, D. Schmitz, M. Schnell, Communication through the phenyl ring: Internal rotation and nuclear quadrupole splitting in p-halotoluenes, 
[8] J. R. Gascooke, W. D. Lawrance, The toluene-Ar complex: S0 and S1

[7] A. Taleb-Bendiab, K. W. Hillig, R. L. Kuczkowski, Microwave spectrum of toluene $\bullet S O 2$ : Structure, barrier to internal rotation, and dipole moment, The Journal of Chemical Physics 98 (5) (1993) 3627-3636. doi:10.1063/ 1.465069 .

Molecular Physics 111 (14-15) (2013) 2189-2197. doi:10.1080/00268976. 2013.796414

van der Waals modes, changes to methyl rotation, and torsion-van der Waals vibration coupling, Journal of Chemical Physics 138 (8) (feb 2013). doi:10.1063/1.4792642.

q [9] D. Schmitz, V. Alvin Shubert, T. Betz, M. Schnell, Multi-resonance

a effects within a single chirp in broadband rotational spectroscopy: The rapid adiabatic passage regime for benzonitrile, Journal of Molecular Spectroscopy 280 (1) (2012) 77-84. doi:10.1016/j.jms.2012.08.001.

口 URL https://wWw.sciencedirect.com/science/article/pii/ S0022285212001488

[10] C. Pérez, A. Krin, A. L. Steber, J. C. López, Z. Kisiel, M. Schnell, Wetting 275 - Camphor: Multi-Isotopic Substitution Identifies the Complementary Roles of Hydrogen Bonding and Dispersive Forces, Journal of Physical Chemistry Letters 7 (1) (2016) 154-160. doi:10.1021/acs.jpclett.5b02541. URL https://pubs.acs.org/doi/10.1021/acs.jpclett. 5b02541https://pubs.acs.org/doi/10.1021/acs.jpclett.5b02541.

[11] G. G. Brown, B. C. Dian, K. O. Douglass, S. M. Geyer, S. T. Shipman, B. H. Pate, A broadband Fourier transform microwave spectrometer based on chirped pulse excitation, Review of Scientific Instruments 79 (5) (2008) 053103. doi:10.1063/1.2919120.

URL http://aip.scitation.org/doi/10.1063/1.2919120

${ }_{285}[12]$ H. M. Pickett, The fitting and prediction of vibration-rotation spectra with spin interactions, Journal of Molecular Spectroscopy 148 (2) (1991) 
371-377. doi:10.1016/0022-2852(91) 90393-0.

口

URL https://www.sciencedirect.com/science/article/pii/ 0022285291903930

[13] H. Hartwig, H. Dreizler, The microwave spectrum of trans-2,3dimethyloxirane in torsional excited states, Zeitschrift fur Naturforschung - Section A Journal of Physical Sciences 51 (8) (1996) 923-932. doi:10.1515/zna-1996-0807. URL https://www.degruyter.com/view/j/zna.1996.51.issue-8/ zna-1996-0807/zna-1996-0807.xml

[14] Z. Kisiel, L. Pszczółkowski, I. R. Medvedev, M. Winnewisser, F. C. De Lucia, E. Herbst, Rotational spectrum of trans-trans diethyl ether in the ground and three excited vibrational states, Journal of Molecular Spectroscopy 233 (2) (2005) 231-243. doi:10.1016/j.jms.2005.07.006.

URL https://www.sciencedirect.com/science/article/abs/pii/ S0022285205001554

[15] Z. Kisiel, Assignment and Analysis of Complex Rotational Spectra, in: Spectroscopy from Space, Kluwer Academic Publishers, 2001, pp. 91-106. doi:10.1007/978-94-010-0832-7_6

[16] F. Neese, The ORCA program system, Wiley Interdisciplinary Reviews: Computational Molecular Science 2 (1) (2012) 73-78. doi:10.1002/wcms. 81.

URL http://doi.wiley.com/10.1002/wcms.81

[17] P. J. Stephens, F. J. Devlin, C. F. Chabalowski, M. J. Frisch, Ab Initio 310 calculation of vibrational absorption and circular dichroism spectra using density functional force fields, Journal of Physical Chemistry® 98 (45) (1994) 11623-11627. doi:10.1021/j100096a001.

URL https://pubs .acs .org/sharingguidelines

[18] D. Rappoport, F. Furche, Property-optimized Gaussian basis sets for 
molecular response calculations, Journal of Chemical Physics 133 (13) (oct 2010). doi:10.1063/1.3484283.

[19] S. Grimme, Semiempirical hybrid density functional with perturbative second-order correlation, Journal of Chemical Physics 124 (3) (2006). doi:10.1063/1.2148954.

[20] F. Weigend, R. Ahlrichs, Balanced basis sets of split valence, triple zeta valence and quadruple zeta valence quality for $\mathrm{H}$ to $\mathrm{Rn}$ : Design and assessment of accuracy, Physical Chemistry Chemical Physics 7 (18) (2005) 3297-3305. doi:10.1039/b508541a.

[21] S. Grimme, S. Ehrlich, L. Goerigk, Effect of the damping function in dispersion corrected density functional theory, Journal of Computational Chemistry 32 (7) (2011) 1456-1465. doi:10.1002/jcc.21759.

URL http://doi .wiley.com/10.1002/jcc.21759

[22] A. D. Becke, E. R. Johnson, A density-functional model of the dispersion interaction, Journal of Chemical Physics 123 (15) (oct 2005). doi:10. $1063 / 1.2065267$.

[23] M. Head-Gordon, J. A. Pople, M. J. Frisch, MP2 energy evaluation by प direct methods, Chemical Physics Letters 153 (6) (1988) 503-506. doi: 10.1016/0009-2614(88)85250-3.

[24] T. H. Dunning, Gaussian basis sets for use in correlated molecular calculations. I. The atoms boron through neon and hydrogen, The Journal of Chemical Physics 90 (2) (1989) 1007-1023. doi:10.1063/1.456153.

[25] R. A. Kendall, T. H. Dunning, R. J. Harrison, Electron affinities of the firstrow atoms revisited. Systematic basis sets and wave functions, The Journal of Chemical Physics 96 (9) (1992) 6796-6806. doi:10.1063/1.462569.

${ }_{340}$ [26] Z. Kisiel, L. Pszczółkowski, E. Białkowska-Jaworska, S. B. Charnley, The millimeter wave rotational spectrum of pyruvic acid, Journal of Molecular Spectroscopy 241 (2) (2007) 220-229. doi:10.1016/j.jms.2006.12.011. 
[27] A. Kraśnicki, L. Pszczółkowski, Z. Kisiel, Analysis of the rotational spectrum of pyruvonitrile up to $324 \mathrm{GHz}$, Journal of Molecular Spectroscopy 260 (1) (2010) 57-65. doi:10.1016/j.jms.2009.12.005

[28] D. Gerhard, A. Hellweg, I. Merke, W. Stahl, M. Baudelet, D. Petitprez, G. Wlodarczak, Internal rotation and chlorine nuclear quadrupole coupling of o-chlorotoluene studied by microwave spectroscopy and ab initio calculations, Journal of Molecular Spectroscopy 220 (2) (2003) 234-241. doi : 10.1016/S0022-2852(03)00125-5.

[29] P. Groner, Effective rotational Hamiltonian for molecules with two periodic large-amplitude motions, Journal of Chemical Physics 107 (12) (1997) 4483-4498. doi:10.1063/1.474810

[30] P. Groner, Effective rotational Hamiltonian for molecules with internal rotors: Principles, theory, applications and experiences, Journal of Molecular Spectroscopy 278 (1) (2012) 52-67. doi:10.1016/j.jms.2012.06.006.

[31] L. B. Favero, L. Evangelisti, G. Feng, L. Spada, W. Caminati, Conformation and internal motions of dimethyl sulfate: A microwave spectroscopy study, Chemical Physics Letters 517 (4-6) (2011) 139-143. doi: 10.1016/j.cplett.2011.10.021.

[32] R. A. Mata, M. A. Suhm, Benchmarking Quantum Chemical Methods: Are We Heading in the Right Direction?, Angewandte Chemie - International Edition 56 (37) (2017) 11011-11018. doi:10.1002/anie.201611308. URL http://doi.wiley.com/10.1002/anie.201611308 3mp van der Waals complexes, Unpublished Results (2019). 